\title{
ASTROPHYSICAL RESULTS FROM GEODETIC VLBI CAMPAIGNS
}

\author{
S. BRITZEN, A. WITZEL and A.-M. GONTIER \\ Max-Planck-Institut für Radioastronomie, Bonn, Germany \\ C.J. SCHALINSKI \\ Institut de Radio Astronomie Millimétrique, Grenoble, France \\ and \\ J. CAMPBELL \\ Geodätisches Institut der Universität Bonn, Germany
}

Geodetic VLBI observations of extragalactic radio sources -designed to measure earth orientation parameters- are performed regularly since more than 10 years now. In different campaigns about 20 Quasars and BL Lacs are being monitored at 2.3 and $8.4 \mathrm{GHz}$. The high duty-cycle of these observations (every month for IRIS-S) and the resulting enormous data base allow to study the milliarcsecond structures and their variations simultaneously at two frequencies on short timescales. Since the accuracy of baseline determination and measurement of earth rotation parameters has reached a limit where source structure contributions become significant, there is substantial interest to monitor and correct for the source structure "effects". Therefore we perform a regular analysis of sources selected from IRIS-S (high north-south resolution and almost circular beam for low declination sources) and EUROPE (high sensitivity due to large telescopes) campaigns covering a maximum time base of 10 years (1983-1993). The (undesired) structure of the sources leads to an occasional updating of the source catalogue in order to have "pointlike" sources which are observed 10-15 times for three to seven minutes during 24 hours. Meanwhile about 50 sources have been observed in geodetic campaigns. The comparatively poor data quality requires careful calibration with a source whose structure is well-known from high dynamic range observations (e.g. the blazar 1803+784). The dynamic range of these maps is limited to 1:50. The merit of this database lies obviously in the monthly persecution of structural changes in extragalactic radio sources over at best 10 years time and the filling of the gaps between high dynamic range observations. Sources which are presently of particular interest to us, include $1803+784,4 \mathrm{C} 39.25$, $0528+134,3 \mathrm{C} 454.3$ and OJ287.

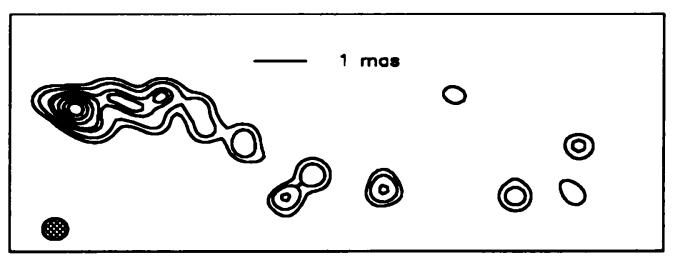

Fig. 1. 8.4 GHz CLEAN map of $1803+784$ (IRIS, May 1988). FWHM of the restoring beam is 0.5 mas. Contours are 5, 10, 20, 25, 40,60 and $80 \%$ of the peak brightness.

423

T. J.-L. Courvoisier and A. Blecha: Multi-Wavelength Continuum Emission of AGN, 423.

(C) 1994 IAU. Printed in the Netherlands. 